Randomised controlled trial

\section{A bottle-weaning counselling intervention for parents of 12-month- old infants reduces bottle use at age 24 months but has no effect on child weight}

10.1136/eb-2014-101825

\section{Li Ming Wen ${ }^{1,2}$}

${ }^{1}$ The 10th People's Hospital, University of Tongji, Shanghai, China; ${ }^{2}$ Sydney Medical School, University of Sydney, Sydney, New South Wales, Australia

Correspondence to: Professor Li Ming Wen, Health Promotion Service, South Western Sydney \& Sydney Local Health Districts, Level 9 North, King George V Building, Missenden Rd, Camperdown, NSW 2050, Australia; liming.wen@sydney.edu.au

Commentary on: Bonuck K, Avraham SB, Lo Y, et al. Bottle-weaning intervention and toddler overweight. J Pediatr 2014;164:306-12.e1-2.

\section{Implications for practice and research}

- A brief educational intervention with parents can reduce the use of bottle feeding for children at 2 years of age.

- The effect of reduced bottle feeding on the early onset of childhood obesity requires further investigation.

\section{Context}

Early infant feeding patterns and practices are modifiable risk factors for childhood overweight and obesity. Prolonged bottle feeding is one such risk factor. Several recent studies have demonstrated the feasibility of bottle-weaning interventions, ${ }^{1-4}$ but the effect of bottle-weaning interventions on reducing childhood obesity is unclear. Bonuck and colleagues study aimed to determine whether brief educational intervention sessions with parents and carers could reduce bottle use and energy intake and by association the risk of infants being overweight at 2 years of age.

\section{Methods}

A randomised controlled trial was conducted with 300 parents of 12-month-old infants who consumed more than two bottles of milk per day. The intervention group received educational sessions delivered by nutritionists during routine women, infants and children nutritional visits every 3 months for a year, as well as being provided with a pamphlet to share with family members. The control group received usual care. Dietary intake and bottle use were assessed via a computer-guided $24 \mathrm{~h}$ recall method. Child length $(\mathrm{cm})$ and weight $(\mathrm{kg})$ were measured by trained researchers at baseline and at 15, 18, 21 and 24 months. Intention-to-treat analyses controlled for baseline measures of outcomes and months post-baseline.

\section{Findings}

At 12-month follow-up the intervention group showed a reduction in bottle use including milk bottles, energy intake from milk bottles and total energy intake. However, the intervention showed no effect on reduction of overweight status; infants $>85$ th centile weight-for-length. The study also found that decreased bottle usage at 15 and 18 months correlated with an increase in 'sippy cup' usage, which could have attenuated the intervention effect on risk of overweight.

\section{Commentary}

There is increasing epidemiological evidence linking infant feeding practices, including shorter duration of breastfeeding, earlier introduction of solid food and other risk behaviours such as physical inactivity and screen time, with the development of obesity in children. ${ }^{2}$ Prolonged bottle feeding is potentially one of the highest risk feeding practices associated with children being overweight.

Bonuck and colleagues should be complimented for their timely study. With increasing recognition of the need to improve infant feeding practices as a means of early childhood obesity intervention, Bonuck's study could be replicable as a model for addressing other risk behaviours, which would have greater public health significance in terms of early obesity prevention. However, it is not surprising that their study found no effect on the overall risk of children becoming overweight with reduced bottle feeding, because it only addressed one risk factor. For an intervention to be effective, multiple risk factors in infant feeding practices need to be intervened. A recent Australian study using a multiple home-visiting model in the first 2 years of life demonstrated significant improvements in meeting recommendations relating to breastfeeding, timing of introducing solid foods and cup usage, ${ }^{3}$ as well as mean BMI reduction among young children. ${ }^{4}$

Given that current infant feeding guidelines advise that infants should be introduced to drinking from a cup at 6 months of age and use of a bottle should be actively discouraged after the age of 1 year, ${ }^{5}$ an intervention starting at 12 months of age may be too late, and ideally, the intervention should be introduced at an earlier age. There is a need for parents to actively encourage the use of a cup at an earlier age, and to discourage the use of bottle feeding. However, whether early cupfeeding could influence children's eating and drinking behaviours or influence the risk of being overweight remains untested. Further studies in this area are greatly needed.

Competing interests None.

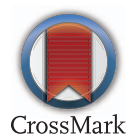

\section{References}

1. Maguire JL, Birken CS, Jacobson S, et al. Office-based intervention to reduce bottle use among toddlers: TARGet Kids! Pragmatic, randomized trial. Pediatrics 2010;126: e343-50.

2. Hesketh KD, Campbell KJ. Interventions to prevent obesity in 0-5 year olds: an updated systematic review of the literature. Obesity 2010;18:S27-35.

3. Wen LM, Baur LA, Simpson JM, et al. Effectiveness of an early intervention on infant feeding practices and 'tummy time': randomized controlled trial. Arch Pediatr Adolesc Med 2011;165:701-7.

4. Wen LM, Baur LA, Simpson JM, et al. Effectiveness of a home-based early intervention on children's BMI at age two years: randomised controlled trial. BMJ 2012;344:e3732.

5. National Health and Medical Research Council. Infant Feeding Guidelines: information for health workers. Canberra: NHMRC, 2012. http://www.nhmrc.gov. au/_files_nhmrc/publications/attachments/n56_infant_feeding_guidelines.pdf 